\title{
Impact of nitrate therapy on the expression of caveolin-1 and its phosphorylated isoform in lungs in the model of monocrotaline induced pulmonary hypertension
}

Original research article/Review

Kmecova Z. ${ }^{凶}$, Malikova E., Zsigmondova B., Radik M., Veteskova J., Marusakova M., Krenek P., Klimas J.

\author{
Comenius University in Bratislava, \\ Faculty of Pharmacy, Department \\ of Pharmacology and Toxicology, \\ Bratislava, Slovak Republic
}

Received 28 February, 2018, accepted 31 May, 2018

Abstract Aim: Nitric oxide signalling pathway showed to be one of the crucial factors in the treatment and pathogenesis of pulmonary arterial hypertension. The aim of this study was to determine the effect of administration of inorganic nitrate, $\mathrm{NaNO}_{3}$, on the expression of caveolin-1 and its phosphorylated isoform (pTyr14Cav-1) in lungs in the experimental model of monocrotaline induced pulmonary hypertension.

Methods: 10 weeks old male Wistar rats were subcutaneously injected with $60 \mathrm{mg} / \mathrm{kg}$ dose of monocrotaline (MCT) or vehicle (CON). Twelve days after the injection, part of the MCT group was receiving $0.3 \mathrm{mM} \mathrm{NaNO}(\mathrm{MCT}+\mathrm{N} 0.3)$ daily in the drinking water and rest was receiving $0.08 \% \mathrm{NaCl}$ solution. Four weeks after $\mathrm{MCT}$ administration, the rats were sacrificed in $\mathrm{CO}_{2}$. Protein expression in lungs was determined by western blot.

Results: We observed a significant decrease in the caveolin-1 expression and a significant shift towards the expression of pTyr14Cav-1 in the group treated with nitrate $(p<0.05)$.

Conclusion: $\mathrm{NaNO}_{3}$ administration affected the expression of caveolin-1 and the ratio of its active (phosphorylated) isoform increased.

Keywords Pulmonary hypertension-monocrotaline-inorganic nitrate-Caveolin-1-lung

\section{INTRODUCTION}

Pulmonary arterial hypertension (PAH) is a rare disease with prevalence and incidence in Europe ranging from 15-60 patients per million and 5-10 patients per million per year, respectively (Galiè et al., 2016). In this disease, endothelial dysfunction and vascular remodelling cause pulmonary vascular resistance and increased pulmonary pressure (Lai et al., 2014). The mean pulmonary arterial pressure is elevated above $25 \mathrm{~mm} \mathrm{Hg}$ at rest, while the pulmonary artery wedge pressure is not higher than $15 \mathrm{~mm} \mathrm{Hg}$ (Galiè et al., 2016). Due to this pressure overload, the right ventricle hypertrophies and failure occurs (Archer et al., 2010).

The median life expectancy in adults without treatment intervention is approximately 2.8 years (Clapp \& Gurung, 2015). However, there are therapeutic approaches that decrease pulmonary pressure, improve right heart function, exercise capacity, quality of life and slow down the progression of the disease (Lai et al., 2014). Specific pharmacotherapy targets three main pathways, affecting vascular tone and vascular remodelling: endothelin-1, prostacyclin and nitric oxide (NO) pathway (Humbert et al., 2004). Currently, endothelin receptor antagonists, prostacyclin analogues, prostacyclin receptor agonists, phosphodiesterase type 5 inhibitors and guanylate cyclase stimulators are used in treatment (Galiè et al., 2016). Despite availability of these therapeutic options, PAH remains incurable (Montani et al., 2014). Therefore, it is vital to look for novel treatment strategies.

Several investigations showed the vasodilatory effect of nitric oxide in the pulmonary circulation and its pathway has been explored for potential therapeutic interventions ever since (Thenappan \&Weir, 2017). NO is synthesized by NO synthases (NOS). There are three isoforms of NOS - endothelial NOS (eNOS), inducible NOS (iNOS) and neuronal NOS (nNOS). NO synthesized by eNOS is thought to be the major source of nitric oxide in lung circulation (Klinger \& Kadowitz, 2017). However, an NOS-independent generation of NO - by reduction from nitrates and nitrites also occurs. Dietary or 
endogenous nitrates require the presence of commensal bacteria in the gastrointestinal tract for its reduction to nitrites, which can be then transformed to NO by various pathways involving myoglobin, haemoglobin, ascorbate, xanthine oxidoreductase or polyphenols (Lundberg et al., 2008).

Caveolin-1 (Cav-1) is a vital structural protein found in the invaginations of the plasmatic membrane - caveolae, and its function is to stabilize several proteins and regulate them through protein-protein interactions (Mathew, 2014). Cav-1 is also important for cellular transport, cholesterol homeostasis (Chettimada et al., 2015), regulation of vascular permeability, cell proliferation and apoptosis (Mathew, 2014). Moreover, Cav-1 inhibits eNOS activity, and therefore NO production, by binding to its domain (Fleming \& Busse, 1999). Phosphorylation on Tyr-14 promotes and enhances the eNOS-Cav-1 binding (Chen et al., 2012).

In this study, we tested the hypothesis that administration of inorganic nitrate could induce changes in eNOS activity through expression of Cav-1 and pTyr14Cav-1.

\section{METHODS}

\section{Experimental Design}

Ten weeks old male Wistar rats were subcutaneously injected either with monocrotaline (Sigma-Aldrich, USA) in dose of $60 \mathrm{mg} / \mathrm{kg}$ (MCT) or vehicle (CON) as in the previous study (Malikova et al., 2016). Twelve days after the application, part of the MCT group started receiving $0.3 \mathrm{mM} \mathrm{NaNO}_{3}$ (CentralChem, Slovakia) (MCT+N0.3) in the drinking water, while the rest was receiving $0.08 \% \mathrm{NaCl}$ solution. Four weeks after MCT administration, the rats were sacrificed by $\mathrm{CO}_{2}$ asphyxiation and the tissue samples from lungs were isolated.

\section{Protein Expression}

The lung samples were homogenized in the presence of liquid nitrogen, centrifuged at $12,560 \mathrm{~g}$ for 15 minutes and then the supernatant was collected. The concentration of samples was determined by BCA method (Pierce BCA Protein Assay, Thermo Fischer, USA). Individual samples were subjected to electrophoresis in polyacrylamide gel. Subsequently, the proteins were transferred to a polyvinylidene difluoride membrane and incubated overnight with the primary antibodies (BD Biosciences, USA) at $4^{\circ} \mathrm{C}$. Afterwards, the membranes were incubated for 1 hour at room temperature with horseradish peroxidase-conjugated secondary antimouse antibody (Jackson ImmunoResearch, UK). For chemiluminescent detection, Pierce ECL Western Blotting Substrate (Thermo Scientific, USA) was used and the density of bands was determined by Optiquant program. The values of bands optical density were normalized by $\beta$-actin values.

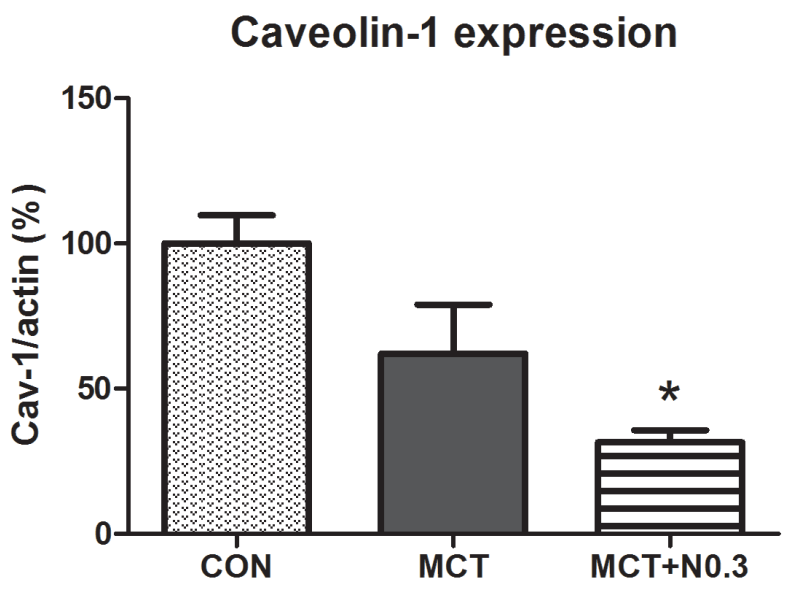

Figure 1. Protein expression of caveolin-1 in lung. Average \pm SEM; $(P<0.05$ vs. CON).

\section{Statistical Analysis}

Acquired data were analysed by the Shapiro-Wilk normality test. Normally distributed data were evaluated by ANOVA, with Tukey's post-hoc test. Non-parametric data were evaluated by Kruskal-Wallis test and Wilcoxon post-hoc test. Results were expressed as mean \pm SEM. $P$ value of $<0.05$ was considered statistically significant.

\section{RESULTS}

Protein expression of caveolin-1 in lung was significantly decreased in MCT+N0.3 compared to the control group ( $P$ $<0.05$ vs. CON) (Fig. 1). We did not observe any significant changes in the expression of phosphorylated isoform of the protein (pTyr14Cav-1); however, there was a non-significant increase in the group treated with nitrate $(P=0.068$ vs. CON) (Fig.2). The ratio pTyr14Cav-1/Cav-1 was significantly increased in MCT+N0.3 ( $P<0.05$ vs. CON) (Fig. 3).

\section{DISCUSSION}

The therapy with $0.3 \mathrm{mM} \mathrm{NaNO}_{3}$ did not prevent an increase of the right ventricular pressure and cardiac hypertrophy induced by monocrotaline (data not shown). However, it affected the expression of caveolin-1 isoforms in lungs. The nitrate-nitrite-NO pathway works with NOS pathway in parallel but, in cases of decreased oxygen availability, the first process is enhanced (Lundberg et al., 2008). We hypothesised that an increased nitrate intake would cause a shift in eNOS activity.

Monocrotaline decreased the caveolin-1 expression in pulmonary arterial endothelial cells (Mathew et al., 2004) 


\section{pTyr14Cav-1 expression}

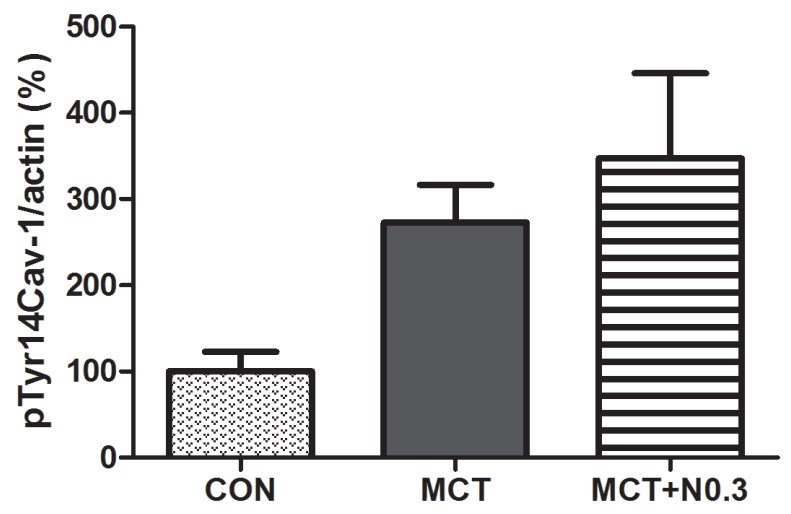

Figure 2. Protein expression of pTyr-14Cav-1 in lung.

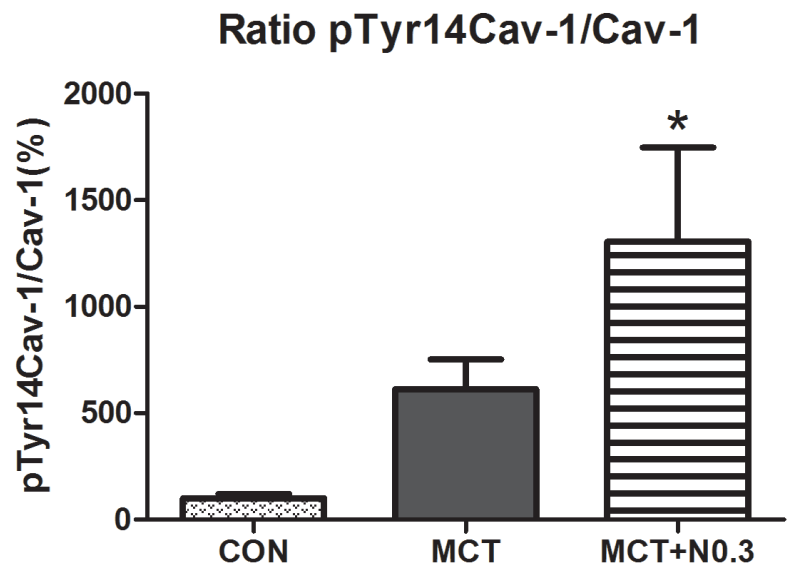

Figure 3. Ratio of pTyr14Cav-1/Cav-1 in lung. Average \pm SEM; ( $P$ $<0.05$ vs. (ON).

and in lung tissue of rats (Haga et al., 2015). We observed non-significant decrease of Cav-1 expression in MCT group as well (by $38 \%, P=0.063$ vs. CON). Zhao et al. demonstrated that knock-out of Cav-1 in mice caused persistent eNOS activity, which led to nitration of protein kinase G (PKG) and subsequently to pulmonary hypertension. Nitration of PKG occurs after NO reacts with superoxide and forms peroxynitrite (Zhao et al., 2009). Under certain pathological states, termed as uncoupling of eNOS, this enzyme is able to produce reactive oxygen species (ROS) such as oxygen radicals (Mathew, 2014).

Moreover, Cav-1 interacts with bone morphogenetic protein receptor type II (BMPR-II). Mutations of BMPR2 gene can be found in 75\% of cases of hereditary PAH (Austin et al., 1993). Cav-1 is necessary for signal transduction and localization of BMPR-II (Wertz \& Bauer, 2008). Cav-1 also inhibits a number of proliferative pathways (Mathew, 2011).

We surprisingly noted even more profound decrease of Cav1 expression in MCT+N0.3 group ( $P<0.05$ vs CON) than in the MCT group. This could suggest a noxious effect of nitrates supplementation, regarding Cav-1. However, there are reports stating that the increase of Cav-1 in pulmonary arterial smooth muscle cells contributes to pathophysiology of PAH (Patel et al., 2007). Regarding this point of view, decrease of Cav-1 might be a positive feature and a compensatory mechanism.

As noted before, Cav-1 inhibits eNOS activity, after phosphorylation on its tyrosine residue (Chen et al., 2012). Moreover, phosphorylation on Tyr-14 is also necessary for the interaction with BMPR-II (Wertz \& Bauer, 2008). We observed a non-significant shift towards the expression of pTyr14Cav-1 in the MCT group and a significant increase of pTyr14Cav-1 expression in the group treated with nitrate $(P<0.05$ vs. CON) This suggests a possibly decreased eNOS activity after nitrate administration. That might be a positive feature, since there is a possibility that the enzyme produces detrimental ROS in the pathological setting of $\mathrm{PH}$. Increase in Cav-1 phosphorylation could also possibly show tendency to enhance signalling through BMPR-II pathway, which was found to be disrupted in PAH (Morrell, 2006).

\section{CONCLUSION}

In conclusion, we demonstrated that $0.3 \mathrm{mM}$ dose of nitrates decreased the expression of caveolin-1 in the lung tissue of monocrotaline-treated rats. However, the ratio of active, phosphorylated form of this protein (pTyr-14Cav-1) and Cav1 significantly increased after the therapy, suggesting an inhibition of endogenous production of NO through eNOScaveolin binding and the potentiation of BMPR-II pathway.

\section{ACKNOWLEGMENT}

The work was supported by the grant APVV-15-0685 Theranostic potential of components of alternative RAS in modulation of right ventricular function and dysfunction from the Slovak Research and Development Agency (Jan Klimas) and grant 1/0294/15 from the Science Grant Agency (VEGA), Slovak Republic (Peter Krenek). 
[1] Archer, S. L., Weir, E. K., \& Wilkins, M. R. (2010). Basic science of pulmonary arterial hypertension for clinicians: new concepts and experimental therapies. Circulation, 121(18), 2045-2066. https://doi.org/10.1161/CIRCULATIONAHA.108.847707

[2] Austin, E. D., Loyd, J. E., \& Phillips, J. A. (1993). Heritable Pulmonary Arterial Hypertension. GeneReviews ${ }^{\oplus}$. University of Washington, Seattle. Retrieved from http://www.ncbi.nlm.nih. gov/pubmed/20301658

[3] Clapp, L. H., \& Gurung, R. (2015). The mechanistic basis of prostacyclin and its stable analogues in pulmonary arterial hypertension: Role of membrane versus nuclear receptors. Prostaglandins \& Other Lipid Mediators, 120, 56-71. https://doi. org/10.1016/j.prostaglandins.2015.04.007

[4] Fleming, I., \& Busse, R. (1999). Signal transduction of eNOS activation. Cardiovascular Research, 43(3), 532-41. Retrieved from http://www.ncbi.nlm.nih.gov/pubmed/10690325

[5] Galiè, N., Humbert, M., Vachiery, J.-L., Gibbs, S., Lang, l., Torbicki, A., ... Luis Zamorano, J. (2016). 2015 ESC/ERS Guidelines for the diagnosis and treatment of pulmonary hypertension. European Heart Journal, 37(1), 67-119. https://doi.org/10.1093/eurheartj/ ehv317

[6] Haga, S., Tsuchiya, H., Hirai, T., Hamano, T., Mimori, A., \& Ishizaka, Y. (2015). A novel ACE2 activator reduces monocrotalineinduced pulmonary hypertension by suppressing the JAK/ STAT and TGF- $\beta$ cascades with restored caveolin-1 expression. Experimental Lung Research, 41(1), 21-31. https://doi.org/10.31 09/01902148.2014.959141

[7] Humbert, M., Sitbon, O., \& Simonneau, G. (2004). Treatment of Pulmonary Arterial Hypertension. New England Journal of Medicine, 351(14), 1425-1436. https://doi.org/10.1056/ NEJMra040291

[8] Chen, Z., Bakhshi, F. R., Shajahan, A. N., Sharma, T., Mao, M., Trane, A., ... Minshall, R. D. (2012). Nitric oxide-dependent Src activation and resultant caveolin-1 phosphorylation promote eNOS/caveolin-1 binding and eNOS inhibition. Molecular Biology of the Cell, 23(7), 1388-1398. https://doi.org/10.1091/ mbc.E11-09-0811

[9] Chettimada, S., Yang, J., Moon, H., \& Jin, Y. (2015). Caveolae, caveolin-1 and cavin-1: Emerging roles in pulmonary hypertension. World Journal of Respirology, 5(2), 126. https://doi. org/10.5320/wjr.v5.i2.126

[10] Klinger, J. R., \& Kadowitz, P. J. (2017). The Nitric Oxide Pathway in Pulmonary Vascular Disease. The American Journal of Cardiology, 120(8), S71-S79. https://doi.org/10.1016/j.amjcard.2017.06.012

[11] Lai, Y.-C., Potoka, K. C., Champion, H. C., Mora, A. L., \& Gladwin, M. T. (2014). Pulmonary arterial hypertension: the clinical syndrome. Circulation Research, 115(1), 115-30. https://doi. org/10.1161/CIRCRESAHA.115.301146

[12] Lundberg, J. O., Weitzberg, E., \& Gladwin, M.T. (2008). The nitratenitrite-nitric oxide pathway in physiology and therapeutics. Nature Reviews Drug Discovery, 7(2), 156-167. https://doi. org/10.1038/nrd2466
[13] Malikova, E., Galkova, K., Vavrinec, P., Vavrincova-Yaghi, D., Kmecova, Z., Krenek, P., \& Klimas, J. (2016). Local and systemic renin-angiotensin system participates in cardiopulmonary-renal interactions in monocrotaline-induced pulmonary hypertension in the rat. Molecular and Cellular Biochemistry, 418(1-2), 147-57. https://doi.org/10.1007/s11010-016-2740-z

[14] Mathew, R. (2011). Cell-specific dual role of caveolin-1 in pulmonary hypertension. Pulmonary Medicine, 2011, 573432. https://doi.org/10.1155/2011/573432

[15] Mathew, R. (2014). Pathogenesis of pulmonary hypertension: a case for caveolin-1 and cell membrane integrity. American Journal of Physiology-Heart and Circulatory Physiology, 306(1), H15-H25. https://doi.org/10.1152/ajpheart.00266.2013

[16] Mathew, R., Huang, J., Shah, M., Patel, K., Gewitz, M., \& Sehgal, P. B. (2004). Disruption of Endothelial-Cell Caveolin-1a/Raft Scaffolding During Development of Monocrotaline-Induced Pulmonary Hypertension. Circulation, 110(11), 1499-1506. https://doi.org/10.1161/01.CIR.0000141576.39579.23

[17] Montani, D., Chaumais, M.-C., Guignabert, C., Günther, S., Girerd, B., Jaïs, X., ... Humbert, M. (2014). Targeted therapies in pulmonary arterial hypertension. Pharmacology \& Therapeutics, 141(2), 172191. https://doi.org/10.1016/j.pharmthera.2013.10.002

[18] Morrell, N. W. (2006). Pulmonary Hypertension Due to BMPR2 Mutation: A New Paradigm for Tissue Remodeling? Proceedings of the American Thoracic Society, 3(8), 680-686. https://doi. org/10.1513/pats.200605-118SF

[19] Patel, H. H., Zhang, S., Murray, F., Suda, R. Y. S., Head, B. P., Yokoyama, U., ... Insel, P. A. (2007). Increased smooth muscle cell expression of caveolin-1 and caveolae contribute to the pathophysiology of idiopathic pulmonary arterial hypertension. The FASEB Journal, 21(11), 2970-2979. https://doi.org/10.1096/ f.07-8424com

[20] Thenappan, T., \& Weir, E. K. (2017). The Nitric Oxide Pathway—A Potential Target for Precision Medicine in Pulmonary Arterial Hypertension. The American Journal of Cardiology, 120(8), S69S70. https://doi.org/10.1016/j.amjcard.2017.06.011

[21] Wertz, J. W., \& Bauer, P. M. (2008). Caveolin-1 regulates BMPRII localization and signaling in vascular smooth muscle cells. Biochemical and Biophysical Research Communications, 375(4), 557-561. https://doi.org/10.1016/J.BBRC.2008.08.066

[22] Zhao, Y.-Y., Zhao, Y. D., Mirza, M. K., Huang, J. H., Potula, H.-H. S. K., Vogel, S. M., ... Malik, A. B. (2009). Persistent eNOS activation secondary to caveolin-1 deficiency induces pulmonary hypertension in mice and humans through PKG nitration. The Journal of Clinical Investigation, 119(7), 2009-18. https://doi. org/10.1172/JCI33338 